\title{
A VELOCITY TRANSFORMATION FOR HEAT AND MASS TRANSFER
}

\author{
F. Nicoud \\ Center for Turbulence Research, Stanford, CA 94305 \\ P. Bradshaw \\ Stanford University, Stanford, CA 94305
}

Published as Physics of Fluids, 12(1):237-238, 2000

\begin{abstract}
The Van Driest transformation for compressible boundary layers with heat transfer is studied in the low Mach number limit. The limiting case leads to a bi-logarithmic type of profile for the mean velocity. A (purely mathematical) analogy with incompressible boundary layers having mass transfer at the wall is identified.
\end{abstract}

The supersonic compressible turbulent boundary layer with or without heat transfer is fairly well documented. ${ }^{1-3}$ Dimensional analysis of the inner layer shows that the law of the wall can be described in terms of two nondimensional wall parameters, the friction Mach number $M_{\tau}=u_{\tau} / c_{w}$ and the heat flux parameter $B_{q}=q_{w} /\left(\rho_{w} C_{p} u_{\tau} T_{w}\right)$, where $u_{\tau}$ is the friction velocity $\sqrt{\tau_{w} / \rho_{w}}, q_{w}$ the heat flux, $C_{p}$ the constant-pressure specific heat, and $T_{w}$ and $c_{w}$ the temperature and speed of sound at the wall. Many experimental and numerical data support the validity of the Van Driest ${ }^{4}$ transformation of the velocity into the form of the incompressible logarithmic law

$$
U_{V D}^{+}=\int_{o}^{u^{+}}\left(\frac{\rho}{\rho_{w}}\right)^{1 / 2} d u^{+}=\frac{1}{\kappa} \ln y^{+}+C
$$

where the additive constant $C$ is in principle a function of both $M_{\tau}$ and $B_{q}$. The superscript '+' denotes the usual wall scaling $U^{+}=U / u_{\tau}$ and $y^{+}=$ $\rho_{w} u_{\tau} y / \mu_{w}$. The case of significant heat transfer but small Mach number has received little attention by experimenters. Therefore the usefulness of the Van Driest transformation to retrieve the classical logarithmic law of the wall cannot yet be satisfactorily demonstrated in this case. However, since the transformation is based on the assumption that turbulence structure is 
unaltered by large temperature variations it should be as valid at low Mach numbers as at high ones.

In this Brief Communication, the behavior of the transformation in the limiting case where $B_{q}$ is finite but $M_{\tau} \rightarrow 0$ is studied, and a mathematical analogy between heat transfer and transpiration is identified. Note that $M_{\tau}=M_{\infty} \sqrt{c_{f} / 2}$ so that for typical "low speed" values of $M_{\infty} \approx 0.05$ and $c_{f} \approx 0.003$ we have $M_{\tau} \approx 0.002$, large enough for round-off errors in numerical work to be negligible.

The "mixing length" formula (obtainable by simple local-scaling arguments without the erroneous mixing length concept) reads, in the fully turbulent region:

$$
\frac{\partial U}{\partial y}=\frac{(\tau / \rho)^{1 / 2}}{\kappa y}
$$

where $\tau$ and $\rho$ are the local shear stress and density. In the case of nonzero $M_{\tau}, U_{\mathrm{VD}}^{+}$can be deduced ${ }^{1}$ as a function of $U^{+}$by integrating the "mixing length' formula in the fully turbulent region, assuming a constant turbulent Prandtl number to derive the temperature and hence the density. We obtain:

$$
U_{V D}^{+} \approx \frac{1}{R}\left[\sin ^{-1}\left(\frac{R\left(U^{+} H\right)}{D}\right)-\sin ^{-1}\left(\frac{R H}{D}\right)\right]
$$

where $R=M_{\tau} \sqrt{\operatorname{Pr}_{\mathrm{t}}(\gamma-1) / 2}, H=B_{q} /\left((\gamma-1) M_{\tau}^{2}\right)$ and $D=\sqrt{C_{1}+R^{2} H^{2}}$.

If Eq. (3) is expanded as a power series in $M_{\tau}$, we obtain:

$$
U_{V D}^{+} \approx \frac{2 \sqrt{C_{1}}}{P r_{\mathrm{t}} B_{q}}\left[1-\sqrt{1-U^{+} \frac{P r_{\mathrm{t}} B_{q}}{C_{1}}}\right]+O\left(M_{\tau}^{2}\right)
$$

Although data for $C_{1}$ are scarce and unreliable, it is reasonable to assume $C_{1} \approx 1 .^{1}$ Then, writing $\mathcal{W} \equiv-P r_{\mathrm{t}} B_{q}$ as the strength of the (not necessarily small) perturbation associated with wall heating or cooling, Eq. (4) can be re-written in the form:

$$
U_{V D}^{+} \approx \frac{2}{\mathcal{W}}\left[\sqrt{1+\mathcal{W} U^{+}}-1\right]+O\left(M_{\tau}^{2}\right)
$$

The first-order term in Eq. (5), i.e. the limit of the Van Driest transformation for low-Mach-number flow with small $M_{\tau}$ but finite $\mathcal{W}$, is formally equivalent to the well-known (e.g. Simpson ${ }^{5}$ ) transformation of the velocity profile for incompressible turbulent wall flows with uniform injection, 
where again $U_{V D}^{+}=(1 / \kappa) \ln y^{+}+C$. In this case, the perturbation parameter is $\mathcal{W}=V_{\mathrm{inj}}^{+}$, the characteristic transpiration velocity being in wall units. Thus, the "mixing length" formulas suggest that an incompressible wall flow subject to injection or suction has the same mean velocity profile as a lowMach-number flow (with variable density) subject to cooling or heating. It must be stressed at once that this curious mathematical analogy is most unlikely to have any simple physical basis.

Consider the $x$-component momentum equation in the two cases, neglecting streamwise gradients. In the flow with heat transfer, we have the familiar constant-stress layer, $\tau=\tau_{w}$, but combining the mixing length formula with constant $P r_{\mathrm{t}}$ gives $\rho_{w} / \rho \approx T / T_{w} \approx 1-P r_{\mathrm{t}} B_{q} U^{+}$. In the transpiration case, the density is constant, i.e. $\rho=\rho_{w}$, but the $x$-component momentum equation, integrated in the $y$ direction, gives $\tau=\tau_{w}+\rho_{w} V_{\mathrm{inj}} U$. In both cases $\tau / \rho$ is of the form $\left(\tau_{w} / \rho_{w}\right)\left(1+\mathcal{W} U^{+}\right)$, and, inserting this into Eq. (2), Eq. (5) follows. If $U_{V D}^{+}$is given by Eq. (1) then $U^{+}$obeys the so-called bi-logarithmic law.

We present this mathematical analogy as a curiosity rather than something with specific physical content, and we make no comment on behavior in the viscous wall region below the validity of Eq. (2); but perhaps investigators of strong heat transfer and of transpiration may find an exchange of views worth while. This note is dedicated to the memory of Prof. Hieu Ha Minh.

\section{References}

[1] P. Bradshaw. Compressible turbulent shear layers. Ann. Rev. Fluid Mech., 9:33-54, 1977.

[2] H.H. Fernholz and P.J. Finley. A critical compilation of compressible turbulent boundary layer data. AGARDOGRAPH 223, 1977.

[3] E.F. Spina, A.J. Smits, and S. Robinson. Supersonic turbulent boundary layers. Ann. Rev. Fluid Mech., 26:287-319, 1994.

[4] E.R. Van Driest. Turbulent boundary layer in compressible fluids. J. Aero. Science, 18(3):145-160, 1951.

[5] R.L. Simpson. Characteristics of turbulent boundary layers at low reynolds numbers with and without transpiration. J. Fluid Mech., 42:769-802, 1970. 